\title{
Culture of microalgae using interstitial water extracted from shrimp pond bottom sediments
}

\begin{abstract}
Interstitial water, extracted from the bottom sediment of a shrimp culture pond, was rich in nutrients containing total phosphorus with concentration of $25.98 \mathrm{mg} / \mathrm{l}$ and total nitrogen of $65.45 \mathrm{mg} / \mathrm{l}$. A diatom, Chaetoceros calcitrans, a green alga, Nannochloropsis oculata, and a cyanobacterium, Oscillatoria sp., were cultured in pure interstitial water (PIW), diluted interstitial water (DIW) and in Conway medium (CM). C. calcitrans showed a significantly higher $(\mathrm{P}<0.05)$ growth rate in DIW compared to CM and PIW. Alternatively, Oscillatoria sp. had a significantly lower $(\mathrm{P}<0.05)$ growth rate when grown in DIW compared to CM. Similar to the other algae, Oscillatoria sp. showed the lowest growth rate in PIW. C. calcitrans and Oscillatoria sp. were also grown in DIW with the addition of nitrogen, phosphorus, silica and the combination of the three nutrients. The addition of nitrogen and silica to the diluted interstitial water significantly improved $(\mathrm{P}<0.05)$ the growth rate of $\mathrm{C}$. calcitrans, but the addition of phosphorus significantly decreased $(\mathrm{P}<0.05)$ its growth. On the other hand, the growth rate of Oscillatoria sp. significantly decreased $(\mathrm{P}<0.05)$ with the addition of nitrogen, but increased $(\mathrm{P}<0.05)$ with the addition of phosphorus. There was no significant difference $(\mathrm{P}>0.05)$ in the growth rate of Oscillatoria sp. cultured in interstitial water and that with added silica or a combination of nitrogen, phosphorus and silica. This study indicates that interstitial water extracted from aquaculture ponds, in sterilized diluted form, has the potential to be used as an effective medium for the culture of microalgae. (C) 2001 Elsevier Science B.V. All rights reserved.
\end{abstract}

Keyword: Interstitial water; Microalgae; Sediment 